This item was submitted to Loughborough's Research Repository by the author.

Items in Figshare are protected by copyright, with all rights reserved, unless otherwise indicated.

\title{
An incremental hybridisation of heterogeneous case studies to develop an ontology for capability engineering
}

PLEASE CITE THE PUBLISHED VERSION

PUBLISHER

INCOSE ( ) Huseyin Dogan, Michael J de C. Henshaw and Julian Johnson

VERSION

AM (Accepted Manuscript)

LICENCE

CC BY-NC-ND 4.0

\section{REPOSITORY RECORD}

Dogan, Huseyin, Michael Henshaw, and Julian Johnson. 2019. "An Incremental Hybridisation of Heterogeneous Case Studies to Develop an Ontology for Capability Engineering". figshare.

https://hdl.handle.net/2134/10212. 
This item was submitted to Loughborough's Institutional Repository (https://dspace.lboro.ac.uk/) by the author and is made available under the following Creative Commons Licence conditions.

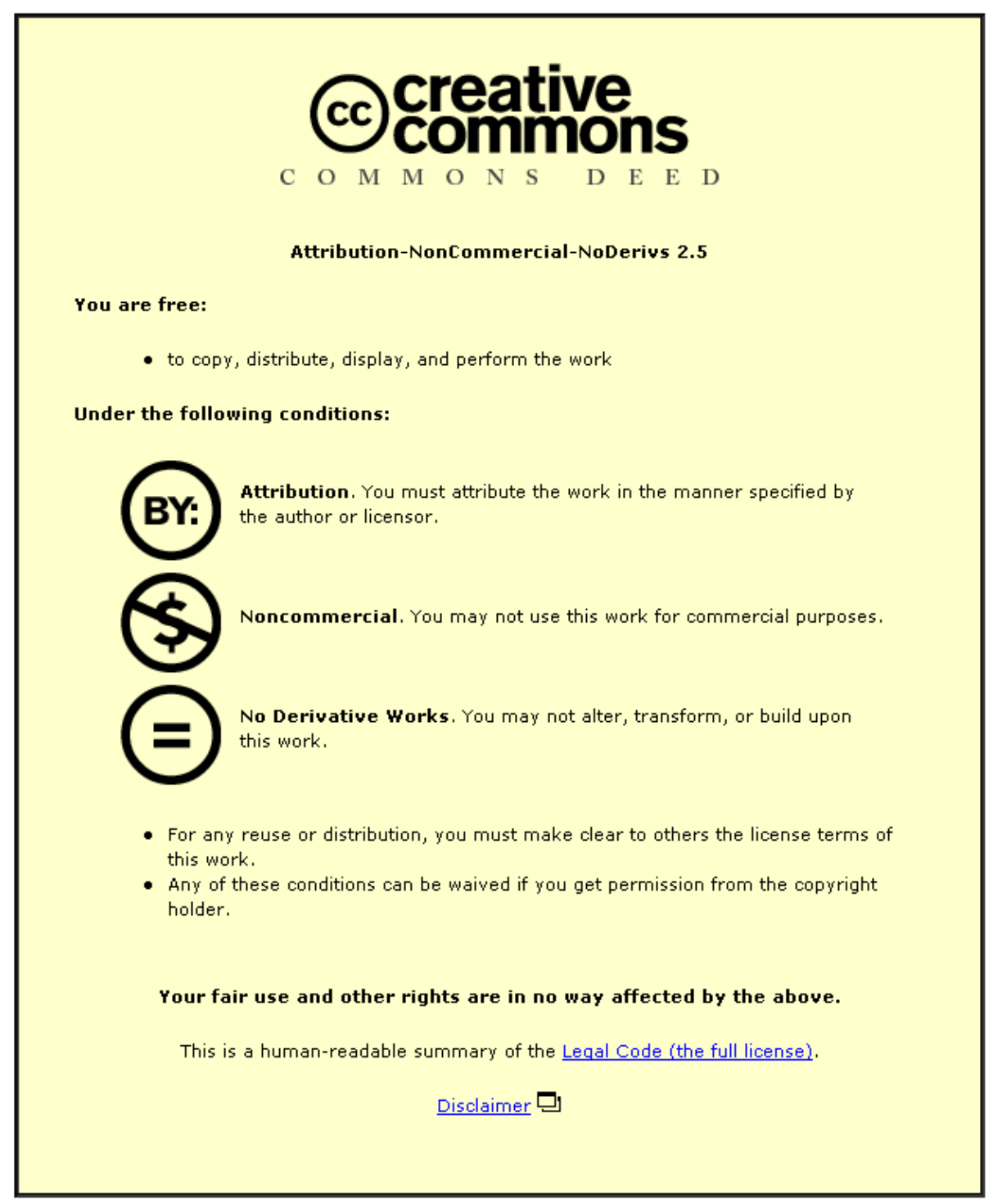

For the full text of this licence, please go to: http://creativecommons.org/licenses/by-nc-nd/2.5/ 


\title{
An incremental hybridisation of heterogeneous case studies to develop an ontology for capability engineering
}

\author{
Huseyin Dogan \\ Engineering Systems of Systems \\ Group \\ Loughborough University, UK
}

\author{
Michael J de C. Henshaw \\ Engineering Systems of Systems \\ Group \\ Loughborough University, UK
}

\author{
Julian Johnson \\ BAE Systems, UK
}

Copyright $\odot 2012$ by Huseyin Dogan, Michael J de C. Henshaw and Julian Johnson. Permission granted to INCOSE to publish and use.

\begin{abstract}
An analysis of perspectives for "capability engineering" has been conducted by the INCOSE UK Capability Working Group (CWG). This paper is a continuation of this study led by the CWG ontology work stream that aims to develop a single shared ontology for the concept of capability engineering to enable semantic interoperability and to support a formal and explicit specification of a shared conceptualisation. Case study material from the different domains of rail, defence and information services was used. The ontology development was executed in three phases; (1) pre-analysis, (2) ontology modelling and (3) post-analysis. The pre-analysis involved literature reviews, requirements specification, systems engineering process utilisation; and resource identification i.e. examination of the case study material. The ontology modelling phase comprised information extraction and classification in addition to modelling and code representation using a mark-up tool, MS Excel and Protégé. The post-analysis involved validation workshops through using expert focus groups.
\end{abstract}

\section{Introduction}

This paper is primarily based on the research conducted by the INCOSE UK Capability Working Group (CWG) which identified eight perspectives of capability and developed an entity relationship diagram for the concept of capability engineering (Henshaw et al. 2010). The CWG perspectives analysis sub-group view 'capability' as the ability to do something which has an overarching approach that links value, purpose, and solution of a systems problem. The holistic thinking mindset, socio-technical nature and similarity in scope to views of systems engineering are emphasised to elaborate the significant difference from product systems engineering and broader characteristics to process perspective of systems engineering.

This paper introduces rigour, richness and detail to the concept of capability engineering thorough development of a user-centred, systematic and case study based ontology. Noy and McGuiness (2000) describe the rationale behind developing an ontology as; to share common understanding of the structure of information to enable semantic interoperability; to enable the reuse of domain knowledge through a data structure and conceptual schema; to make domain assumptions explicit; and to analyse domain knowledge. Semantic interoperability is the ability to exchange data in order to improve interoperability between systems. The scope of capability engineering is large and there are challenges in producing an all-embracing model with a vocabulary agreed by all parties. Modelling the whole space in one set of a language can be difficult. A case study based information extraction approach that looked at 
the problem space in chunks (domains) is described within this paper. The progressive updating of the ontology due to evolving nature of the problem space is discussed by using the initial validation results from the focus groups.

\section{Objectives}

A set of high level objectives are derived through analysis of the CWG workshop discussions and prior meetings with the CWG ontology work stream members:

- Objective 1: to develop a sector-independent ontology by extracting and classifying information from case studies across heterogeneous domains to explicitly specify the concept of capability engineering.

- Objective 2: to develop an information model from the ontology that is to be informed by the capability engineering activity model to support the 'management of knowledge' within the context of capability engineering.

- Objective 3: to evaluate (verify and validate) the ontology through expert reviews and application to a case study.

The next section discusses the current ontology-based approaches and proposes a flow chart for the development of the capability engineering ontology.

\section{Ontology-Based Approaches}

Ontology-based modelling for information and knowledge management has been widely used (Hughes et al. 2009; Duan et al. 2009; Dongmin et al. 2010; Liu et al. 2010). Generally, an ontology is defined as an explicit specification of a conceptualisation where concepts and their relations are extracted from the real world (Studer et al. 1998; Duan et al. 2009). An ontology-based approach is complementary to more conventional modelling approaches such as Unified Modelling Language (UML) and Systems Modelling Language (SysML) because an ontology is significantly useful in defining a domain from multiple author perspectives and terminologies. Allemang 2008 elaborate one of the tenets of the Semantic web "AAA; Anyone can say Anything about Any topic". In contrast to conventional modelling, ontology model has formalisms and associated inferencing that facilitate bringing multiple user perspectives and vocabularies together. However, like conventional modelling, it also focuses on the key concepts, i.e. "what is", as in, "what is capability engineering and how is it different from other engineering domains?". The classes and relationships typically associated with the model can also be added.

A single shared ontology for capability engineering can enable semantic interoperability and support a formal and explicit specification of a shared conceptualisation. As recommended by Unshold and Gruniger (2004) when developing a practical single shared upper ontology, the mapping of ontology amongst domains and the eight different worldviews identified by the INCOSE UK CWG (e.g. equipment, organisational and service centric worldviews) needs to be human assisted rather than fully automated to achieve the interoperability, interconnectedness and correlation desired between these heterogeneous domains. The ontology shall also form tight definitions and be independent of industry sector and applications. These are discussed later within the requirements analysis section.

A variety of methods, methodologies, tools and languages for ontology development have already been analysed by various authors (Corcho et al. 2002; Mizoguchi 2003; Mizoguchi 
and Kozaki 2009). Languages such as OWL (Web Ontology Lanaguage) and IDEF5 (Integrated Definition for Ontology Description Capture Method) in addition to tools including Protégé and Hozo are examined to check their applicability. A process flow chart is also proposed for developing an ontology for capability engineering as a result of analysing a variety of developments including

- ontology-based conceptual knowledge representation model (Kourlimpinis et al. 2008);

- ontology-based information model development for science information reuse and integration (Hughes et al. 2009);

- ontology-based knowledge modelling framework for intangible cultural heritage (Tan et al. 2009);

- domain ontology life-cycle engineering framework for modular product design (Duan et al., 2009; Liu et al. 2011); and

- $\quad$ ontology for product-service system by Cranfield University (Annamalai et al. 2010).

Annamalai et al. (2010) discusses methodologies for developing an ontology through analysing various approaches and argued that "there is no one correct way to model a domain and that ontology development is necessarily an iterative process". Although there are major similarities with these reviewed approaches, the details and context vary. As illustrated in Figure 1, a process flow chart is proposed that incorporates the findings from the above to enhance ontology development through pre-analysis, modelling and post-analysis phases.

The pre-analysis phase predominantly focuses on case studies. These are discussed next.

\section{Case Studies}

\section{Appropriateness of case study material}

Case studies are relatively important when extracting the necessary information and evaluating the ontology developed. Post-analysis evaluation through application to a specific case study can support the validation and refinement of the ontology. The INCOSE UK Capability Working Group was approached to identify the appropriate case studies in heterogeneous domains including defence, rail and information services; progress is reported on these domains herein and further analysis is being conducted in other domains. A list of features that makes the case studies appropriate is derived to guide the decision on case study selection.

- Does the case study contain sufficient material of relevance to our definition of capability (i.e. ability to do something) and can we map this onto the capability engineering entity relationship diagram?

- Which domain does the case study belong to e.g. defence (land/air/sea), rail, health?

- How can we determine the level of abstraction the case study material address e.g. generic domain, collaborative programme, specific project?

- Is there a concise but substantial source that provides detailed information about this case study?

- Is this case study document in a form (e.g. MS Word) that is easy to process?

- What worldviews of the CWG white paper does this case study address?

- What will be the applications of an ontology for this particular case study? 


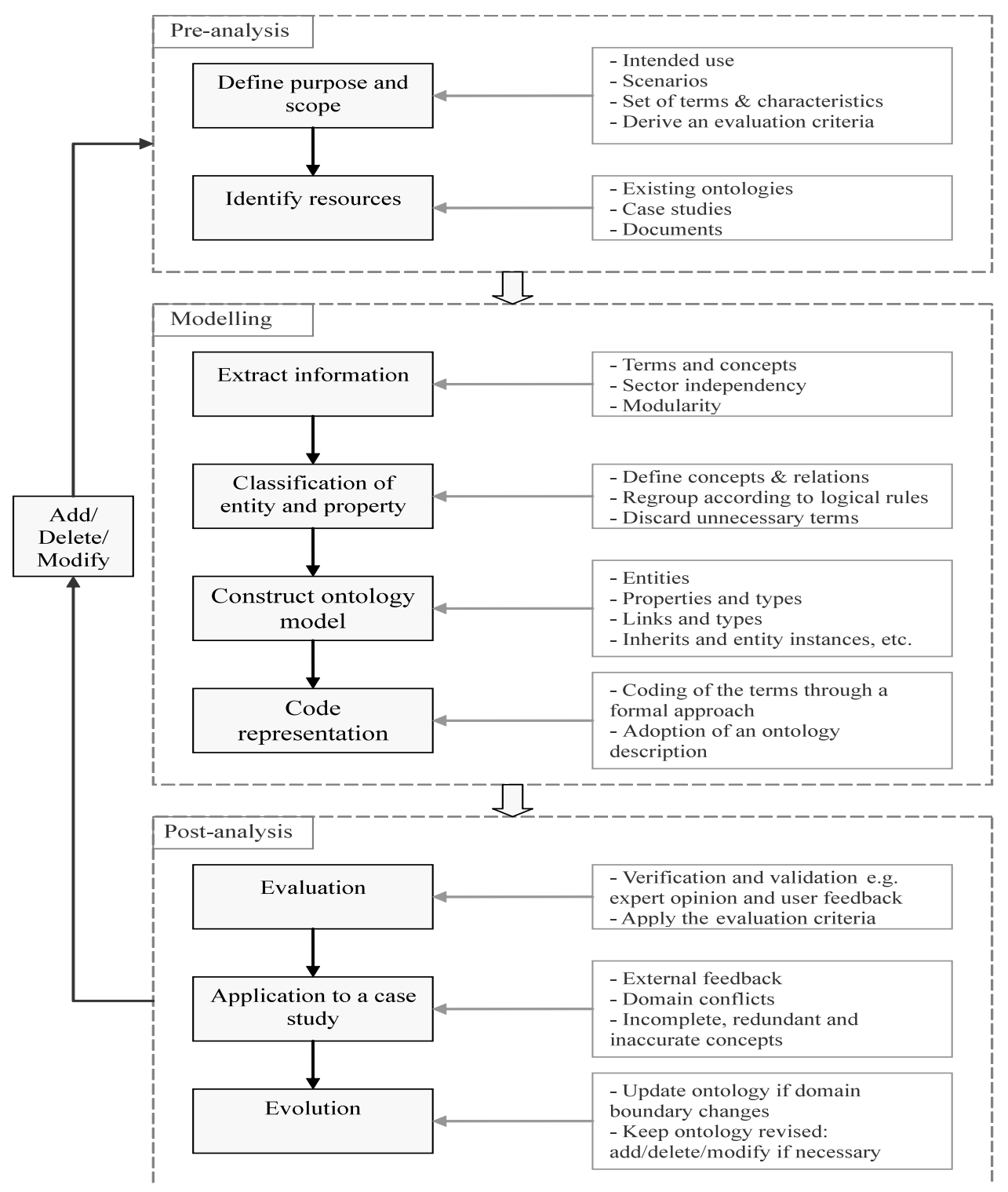

Figure 1. Process flow chart for ontology development

As shown in Figure 2, various case study options have been analysed to determine their relevance to developing a capability engineering ontology. For example, the Queen Elizabeth Class (QEC) ${ }^{1}$ contributes to a high level capability (i.e. the carrier strike capability) and can have capabilities within itself (UKMoD 2012). The case studies in Figure 2 are discussed with regards to their relevance to capability engineering e.g. the search and rescue contribute to the overall crisis management capability but on its own it is very specific. Consequently, this may influence the information resources to be used to extract the terms or concepts. These programmes are perceived as capabilities; QEC is perceived as equipment-based, ATTAC (Availability Transformation: Tornado Aircraft Contract) as service and support related and GVA (Generic Vehicle Architecture) as capability systems engineering.

\footnotetext{
${ }^{1}$ Note material associated with QEC and Carrier Strike in this paper are limited to that available in the public domain.
} 


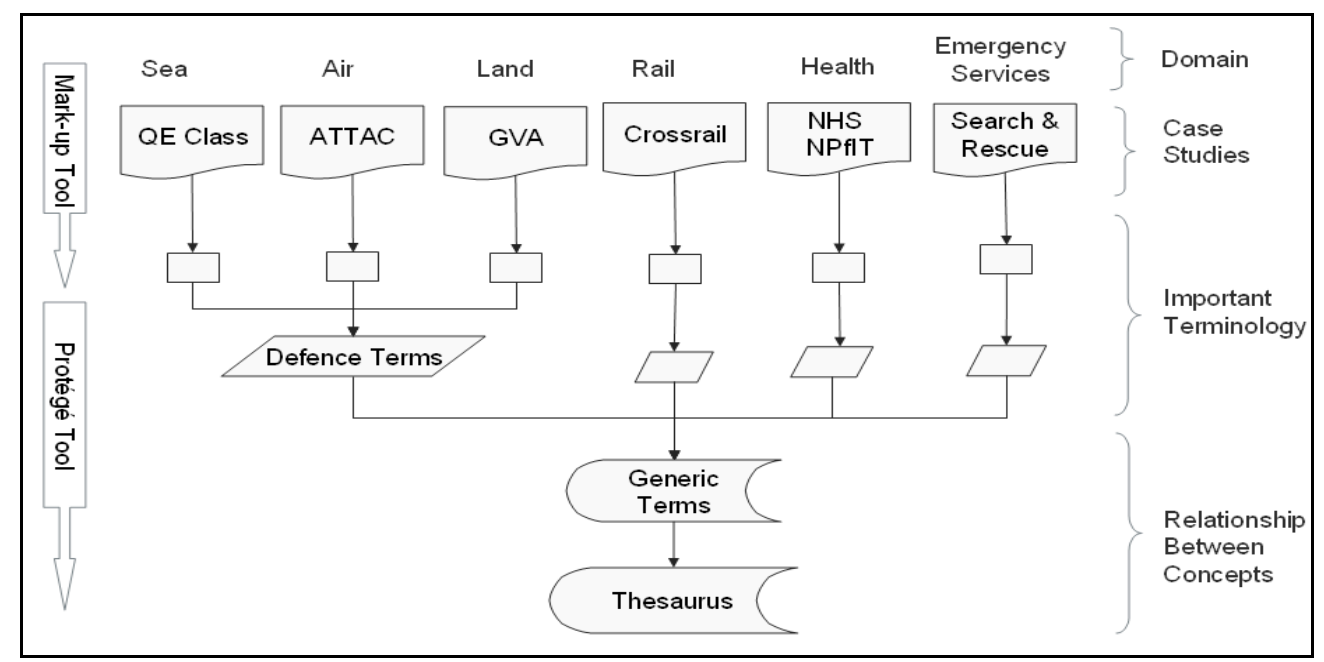

Figure 2. Case study options

A mark-up tool is used to extract important terminology from the case studies and Protégé to construct the ontology i.e. concepts and their relationships.

\section{Case study material as related to the worldviews of Capability}

Material related to the following case studies are obtained and analysed. Examples from these case studies are used to explore and illustrate their relation to the worldviews (Ws) of capability (Table 1) as described by the INCOSE UK Capability Working Group (Henshaw et al. 2010).

Where a particular workview is not seen to be applicable to a particular case study, that worldview is marked "N/A".

Table 1: Summary of the Worldviews (Ws) extracted from Henshaw et al. (2010)

\begin{tabular}{|l|l|l|}
\hline W1. & $\begin{array}{l}\text { Equipment } \\
\text { Capability }\end{array}$ & $\begin{array}{l}\text { Defining the needs of users against which suppliers design and develop } \\
\text { equipment that has capability. }\end{array}$ \\
\hline W2. & Capability Planning & $\begin{array}{l}\text { Capability is used to translate a set of explicit user wants into a set of solution } \\
\text { independent requirements. }\end{array}$ \\
\hline W3. & $\begin{array}{l}\text { Capability } \\
\text { Trade-off }\end{array}$ & $\begin{array}{l}\text { Continually and continuously determining capability needs and funds available } \\
\text { to design a programme to decide and balance in which capability to invest. }\end{array}$ \\
\hline W4. & Service Capability & $\begin{array}{l}\text { Defining, developing, and using specific business services continuously in } \\
\text { addition to developing and storing fallback services to be used at a future date. }\end{array}$ \\
\hline W5. & $\begin{array}{l}\text { Dynamic Capability } \\
\text { Reconfiguration }\end{array}$ & $\begin{array}{l}\text { Reconfiguring available assets, people and processes quickly to meet current } \\
\text { circumstances. }\end{array}$ \\
\hline W6. & $\begin{array}{l}\text { Capability Systems } \\
\text { Engineering }\end{array}$ & $\begin{array}{l}\text { Developing and operating a capability solution across (and incorporating) all } \\
\text { contributing components of capability (CoC). }\end{array}$ \\
\hline W7. & Enterprise Planning & $\begin{array}{l}\text { Developing and maintaining an integrated plan to manage the interdependencies } \\
\text { between all CoC changes across all capabilities and business services. }\end{array}$ \\
\hline W8. & $\begin{array}{l}\text { Organisational } \\
\text { Capability }\end{array}$ & $\begin{array}{l}\text { Capability is based on the resources available to an organisation and also } \\
\text { emerges through processes of interaction between individuals, groups and } \\
\text { organisations. }\end{array}$ \\
\hline
\end{tabular}

Rail Value for Money (RVfM) - Whole Systems Programme Management. This is a report written by Atkins Ltd. commissioned by RVfM team to develop whole systems programme management into practical approaches that reduce all aspects of cost associated with delivery of major projects within rail in UK (e.g. Crossrail, Thameslink) 
(Elphick 2011). The following examples illustrate the worldviews as associated to this rail case study.

W1. Equipment Capability - National Rail (NR) specifies and develops or procures infrastructure assets (signalling, new stations etc.), Department for Transport (DfT) specifies and procures new rolling stock.

W2. Capability Planning - A set of explicit user wants e.g. "delivering 50K passengers an hour in the peak" is translated into a written set of solution independent requirements and systems design options to satisfy the capability needs e.g. 24 trains per hour, new signalling, automatic train operation, new rolling stock and new franchises.

W3. Capability Trade-off - The Secretary of State for Transport continually and continuously determines the whole life assurance and design options for rail capability needs and funds available to decide on programmes such as Thameslink and Crossrail to deliver systems to meet capability needs that include e.g. delivering 24 trains per hour or a new cross-London service.

W4. Service Capability - Specific business services such as 24 trains per hour and reduced overcrowding are delivered though a programme sponsored by DfT; capability components such as equipment (e.g. new trains) and (infrastructure e.g. longer platforms) are transitioned into a service that is to be operated and continuously improved in line with the strategic plans of the DfT.

W5. Dynamic Capability Reconfiguration - Fallback timetables are produced and Network Rail and the train operating company will occupy a single operations centre to facilitate effective disruption management.

W6. Capability Systems Engineering - A capability solution such as Crossrail "linking east and west London through a 13 mile tunnel under central London providing direct access to the centre of London without change of mode" is to be developed and operated by an enterprise of users and suppliers including Network Rail, London Underground etc through incorporating all components of capability from equipment (e.g. new trains) to infrastructure (e.g. twin bore tunnel under central London) and deploying all appropriate systems engineering approaches and techniques throughout its lifecycle from concept to disposal.

W7. Enterprise Planning - N/A.

W8. Organisational Capability -N/A

Queen Elizabeth (QE) Class Development in the context of Carrier Strike (CS). The Queen Elizabeth Class (QEC) carrier operating with Joint Combat Aircraft (JCA), the most modern combat jets, will give the UK the ability to project military power more than 700 nautical miles over land, as well as sea, from anywhere in the world (UKMOD 2012). Case study material previously used to inform the activity model reported in (Touchin 2010) was also analysed in this ontology work. The following examples illustrate the worldviews as associated to the carrier strike case study.

W1. Equipment Capability - UK Ministry of Defence (MoD) has an outline Carrier Strike (CS) concept and created user requirement and system requirement documents for each equipment separately. Suppliers design and develop equipment such as QE class, Joint 
Combat Aircraft (JCA) i.e. JSF, and the Maritime Airborne Surveillance and Control (MASC) (UKMOD 2012); carriers and aircraft on their own can be perceived as equipment that has capability.

\section{W2. Capability Planning - N/A}

W3. Capability Trade-off - MoD determines funds available and a programme is designed to deliver systems to meet the capability needs. Investment is influenced by political and commercial constraints and environment. An example of a capability trade-off follows the Strategic Defence and Security Review (SDSR) in 2010 where the Harrier aircraft is to be withdrawn from service by April 2011.

W4. Service Capability - MoD as the service recipient requires carrier strike capability to project UK Air Power from a main operating base, deployed operating base or the sea. (UKMOD 2012). This capability is achieved by the providers, users and functional responsible person defining the service, transitioning capability components (e.g. supporting communication infrastructure, training of ship's crew) into a service in addition to operating and continuously improving the service, in line with MoD's operating conditions.

\section{W5. Dynamic Capability Reconfiguration - N/A}

W6. Capability Systems Engineering - An enterprise of users and suppliers including the MoD and industry develop and operate carrier strike capability solution (that aims to project UK Air Power on an expeditionary basis) across all contributing components of capability from equipment to infrastructure (i.e. JCA integration with the ship, dockyard developments) by deploying all appropriate systems engineering approaches and techniques from cradle to grave.

\section{W7. Enterprise Planning - N/A}

W8. Organisational Capability -N/A

The National Programme for Information Technology (NPfIT) in the National Health Service (NHS). This is a report produced by National Audit Office for presentation to the House of Commons (NAO 2006). NPfIT is a "ten year programme which presents an unprecedented opportunity to use Information Technology (IT) to reform the way the NHS in England uses information, and hence to improve services and the quality of patient care". Following examples illustrate the worldviews as associated to NPfIT.

\section{W1. Equipment Capability - N/A}

W2. Capability Planning - A set of requirements from the National Health Service (NHS) is translated into a programme that use Information Technology (IT) to reform the way the NHS in England uses information to improve services and the quality of patient care; the suppliers generated systems to satisfy this need that is defined and constrained by the context in which the equipment is used (e.g. choose and book for GPs).

W3. Capability Trade-off - N/A 
W4. Service Capability - A service delivery programme to enable the NHS to become more effective in treating patients i.e. ensuring that accurate patient records are available at all times.

W5. Dynamic Capability Reconfiguration - N/A

W6. Capability Systems Engineering - N/A

W7. Enterprise Planning - N/A

W8. Organisational Capability -N/A

Combat Search and Rescue (CSAR). This case study material is extracted from a $\mathrm{PhD}$ thesis that evaluated SoS against mission requirements. Johnson (2009) defined CSAR as a specific task performed by rescue forces to effect the recovery of assets (including humans, platforms and data) isolated in hostile territory. The following examples illustrate the associated worldviews.

W1. Equipment Capability - N/A

W2. Capability Planning - N/A

W3. Capability Trade-off - N/A

W4. Service Capability - N/A

W5. Dynamic Capability Reconfiguration - Rescue forces deliver search and rescue mission to recover assets isolated in hostile territory through designing a "rescue package" made up of various systems (e.g. helicopter, rescue coordination centre) formed dynamically in a short response time, in line with the CSAR mission.

W6. Capability Systems Engineering - The defence enterprise that consists of the MoD, suppliers etc operate a CSAR solution across (and incorporating) all components of capability (equipment, processes, people etc) by developing systems engineering approaches/techniques to understand the CSAR mission requirements and manage systems (i.e. helicopters, inertial navigation systems) from cradle to grave.

W7. Enterprise Planning - N/A

W8. Organisational Capability -N/A

The INCOSE UK CWG white paper on capability engineering perspective analysis (Henshaw et al. 2010) is also used to support the completeness of the ontology as the white paper outlined further concepts through Soft Systems Methodology (SSM) root definitions.

\section{Requirements Analysis}

This section reports on the requirements of the overall study. This involves employing an iterative requirements analysis approach to capture, analyse, and synthesise the key stakeholder requirements. Since this is not a product or software development project, the requirements analysis study used techniques from a synthesis of approaches including the Quality Function Deployment (QFD), Unified Modelling Language (UML) and the Volere Requirements Specification (VRS). Systemic Textual Analysis (STA) and VRS are used to develop an unambiguous, testable and traceable set of requirements. The STA pro-forma layout helps to identify missing requirements and is therefore incorporated into the VRS template (Volere 2011). In addition, an iterative PDCA (plan-do-check-act) process is employed to manage the requirements i.e. elicit, capture and validate the expectations of the stakeholders through a series of stakeholder interactions. 
The assumptions, constraints, non-functional requirements, functional requirements and project issues are extracted using STA through analysing previous documents and proposals including the INCOSE UK CWG workshop, white paper and presentations; and also the academic research group discussions. The requirements specification pro-forma listing these requirements is extracted from the hybrid of STA and VRS template as shown in Figure 3.

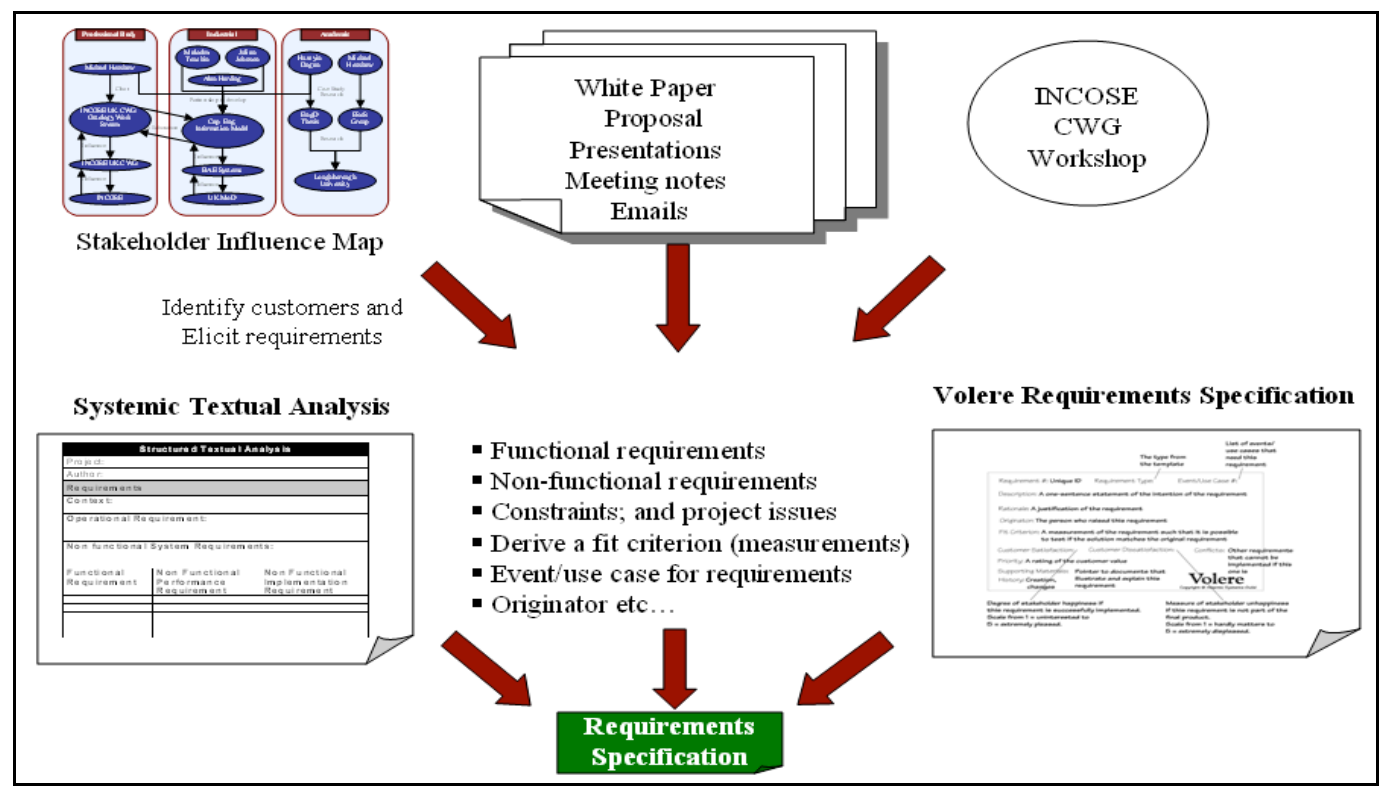

Figure 3. Requirements Analysis process adopted: STA and VRS.

A stakeholder influence map was created to understand the interactions of stakeholders. The stakeholders are divided into three groups; professional body, industrial and academic. A requirements specification was also generated and iteratively updated as a result of user feedback. An extract from the requirements specification table is shown in Figure 4. This includes a list of functional requirements (FR); non-functional requirements (NFR); and also project issues and constraints (PIC). A fit criterion for each requirement is created to test whether the requirement was in fact implemented as specified in the project. The requirements are also mapped back to the use cases developed to enable traceability and can easily be correlated to the set of high level objectives as described in the introduction section.

\begin{tabular}{|c|c|c|}
\hline Type & Description & Fit Criterion/Measurement \\
\hline NFR 1 & The ontology shall be sector-independent. & $\begin{array}{l}\text { Terms and concepts cross-checked from different } \\
\text { sectors for generalisation. }\end{array}$ \\
\hline NFR 2 & The ontology shall be applic ation-independent. & $\begin{array}{l}\text { Use a standard ontology language i.e. OWL and } \\
\text { test on various software. }\end{array}$ \\
\hline NFR 3 & $\begin{array}{l}\text { The ontology shall enable semantic interoperability and transition } \\
\text { between heterogeneous domains and worldviews. }\end{array}$ & $\begin{array}{l}\text { Description of generic terms and concepts; } \\
\text { application to a case study; expert reviews. }\end{array}$ \\
\hline NFR 4 & The ontology shall form tight definitions. & $\begin{array}{l}\text { Check mutually consistencies of ontology i.e. } \\
\text { crosscheck concepts and definitions. }\end{array}$ \\
\hline NFR 5 & $\begin{array}{l}\text { The ontology shall extract, classify and describe the key terms } \\
\text { and concepts. }\end{array}$ & $\begin{array}{l}\text { Use a standard language (i.e. OWL) and software } \\
\text { (i.e. Protégé) to enable this. }\end{array}$ \\
\hline FR 16 & $\begin{array}{l}\text { The ontology will be realised through a prototype that will } \\
\text { respond to queries; enable updates and user interaction. }\end{array}$ & User evaluation thorough a set of tasks. \\
\hline PIC 17 & The ontology outputs shall be made public. & Publications - conference/journal papers. \\
\hline
\end{tabular}

Figure 4. Extract from the requirements specification table. 
The uses of the ontology, when supported by appropriate tools and applications, can be summarised as:

- support collaboration within and between domains through provision of common terminology;

- enable improvement of CEAM (Capability Engineering Activity Model) using robust definitions

- enable improvement of SOSA-AM (Systems of Systems Approach - Activity Model) using robust definitions;

- enable assessment of enterprise architecture frameworks for use in capability engineering by comparison with their meta models;

- act as an index or viewpoint onto information and knowledge related to capability engineering and wider systems engineering; and also

- contribute (in an appropriate manner) to BKCASE (Body of Knowledge and Curriculum to Advance Systems Engineering) definitive view of capability engineering.

Following the summary of case studies and the requirements analysis process, we focus on discussing the ontology development process and initial results.

\section{Ontology Development}

This section exploits the process flow chart illustrated in Figure 1 to develop an ontology. The pre-analysis, modelling and post-analysis phases are described further.

\section{Pre-analysis}

The pre-analysis involved a literature review, requirements analysis and resource identification as described earlier. Examination of the case studies, reports and documents to check their relevance and richness for information extraction has already been discussed. Existing ontologies and approaches e.g. ontology for product-service system (Annamalai et al. 2010) have been analysed to build a process flow chart that encapsulated a procedure for ontology development.

\section{Ontology modelling}

The ontology modelling phase involved information extraction and classification. This process includes extraction of concepts form the resources available; classification of entities and properties according to logical rules; and also model and code representation using Protégé to illustrate the results. Protégé is "a free, open-source platform that provides a growing user community with a suite of tools to construct domain models and knowledge-based applications with ontologies" (Protégé 2011). The subject-predicate-object clauses of RDF (Resource Description Framework) known as "n-triples" (Allemang and Hendler 2008) are also used to show the relationships between concepts.

Term extraction. A mark-up tool was used to highlight the key terms also referred to as individuals or instances from the case study material. These were then copied into a text file and imported into MS Excel for further analysis. The Entity Relationship Diagram (ERD) from the INCOSE CWG perspective analysis paper was used as a baseline to provide a classification as illustrated in Figure 5. 


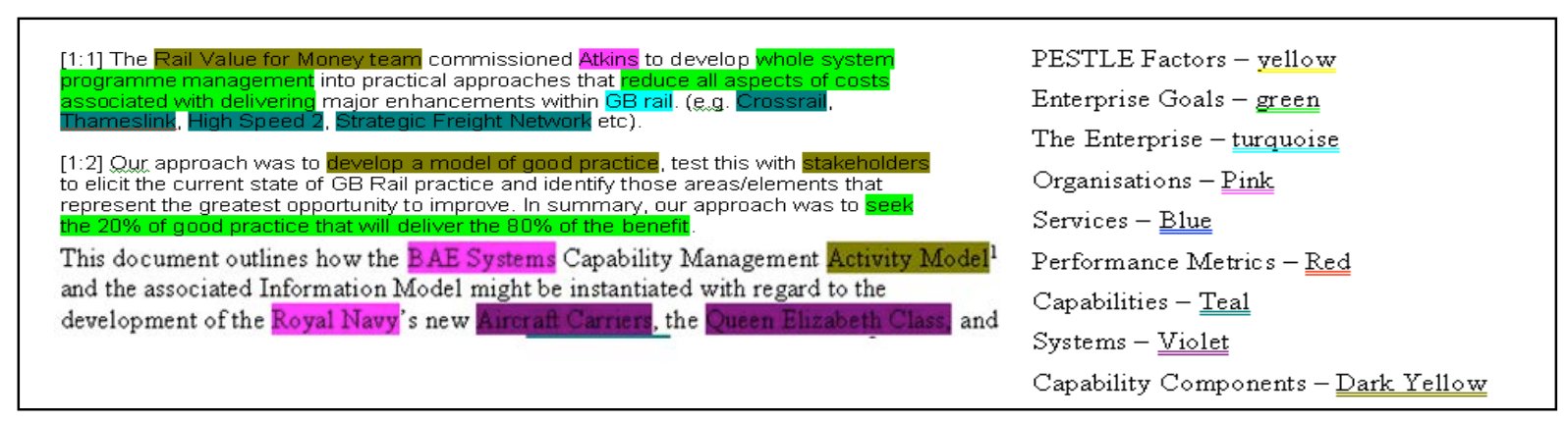

Figure 5. Term extraction using the mark-up tool and pre-determined classification.

Term analysis and n-triples generation. This task involved importing the terms or concepts extracted through the mark-up tool into MS Excel for further analysis. The analysis comprised subsequent grouping of these terms into more general concepts; generating statements showing the relationship and context of use of the terms; adding new concepts that are not capture by the ERD classification; and also generating RDF n-triples by using the subject-predicate-object expressions to denote the relationships. Figure 6 shows some examples of n-triples derived from the CWG perspective analysis case study. A total of 157 n-triples were created for INCOSE CWG perspective analysis case study on its own.

\begin{tabular}{|c|c|c|c|c|}
\hline$\#$ & Subject & Predicate & Object & rdfs statement \\
\hline 1 & EnvironmentalFactor & affects & EnterpriseGoal & :EnvironmentalFactor : affects : EnterpriseGoal. \\
\hline 2 & CapabilityComponent & has & Interdependency & CapabilityComponent :has :Interdependency. \\
\hline 3 & Enterprise & consistsOf & Organisation & Enterprise : consistsOf : Organisation. \\
\hline 4 & Capability & realisedThrough & System & Capability :realisedThrough : System. \\
\hline 5 & MilitaryOrganisation & subClassOf & Organisation & MilitaryOrganisation rdfs: subClassOf : Organisation. \\
\hline 6 & Capability & consistsOf & Resources & Capability :consistsOf :Resources. \\
\hline 7 & ServiceProvider & delivers & Business Service & ServiceProvider : delivers :Business Service. \\
\hline 8 & Capability & realisedThrough & Services & :Capability :realisedThrough :Services. \\
\hline 9 & EquipmentCapability & subClassOf & Capability & EquipmentCapability rdfs: subClassOf : Capability. \\
\hline 10 & CapabilityTradeOff & subClassOf & Capability & CapabilityTradeOff rdfs: subClassOf : Capability. \\
\hline 11 & Capability & hasMultiple & Perspectives & Capability :hasMultiple :Perspectives. \\
\hline 12 & Capability & generates & Outcome & Capability :generates :Outcome. \\
\hline 13 & Capability & requires & Planning & Capability :requires :Planning. \\
\hline 14 & Strategist & determines & CapabilityNeed & Strategist :determines :CapabilityNeed. \\
\hline 15 & Strategist & decidesOn & Investment & Strategist : decidesOn :Investment. \\
\hline 16 & Capability & builtFrom & CapabilityComponent & Capability :builtFrom :CapabilityComponent. \\
\hline 17 & CapabiltyEngineering & includes & HolisticThinking & CapabiltyEngineering : includes : HolisticThinking. \\
\hline 18 & CapabilityEngineering & is $\mathrm{An}$ & Activity & CapabilityEngineering : is An :Activity. \\
\hline
\end{tabular}

Figure 6. Examples of RDF n-triples: subject-predicate-object clauses.

Model representation. Focus groups were used to validate the RDF n-triples. The participants, who were knowledgeable about the subject area, pursued the following procedure to sketch an initial ontology for capability engineering: (1) eliminate some of the subject-predicate-object clauses; (2) refine the final list i.e. check consistency and meaning; (3) use the "subClassOf" predicate or relationship to build a hierarchy; and (4) use the remaining subject-predicate-object clauses to build the initial cut of the capability engineering ontology.

The focus group results were then copied across into Protégé for ontology generation and post-analysis which is discussed in the next section.

\section{Post-analysis}

The post-analysis phase is concerned with ontology evaluation and therefore comprises the validation of the ontology through expert reviews, user feedback and application to a case 
study to check its suitability. The evolution of the ontology is significantly important as procedures need to be put in place for keeping the ontology revised and up to date i.e. adding, deleting or modifying the ontology to incorporate boundary changes.

The outcome of the three phases resulted in the initial ontology as discussed in the next section.

\section{Analysis of initial results}

The term extraction results for three of the case studies are summarised in Figure 7. In total, there are 512 terms extracted with 406 n-triples. The NPfIT and CSAR case studies are parked to be used when evaluating the finalised ontology. The n-triples are exploited in the focus groups to construct an initial ontology for each case study.

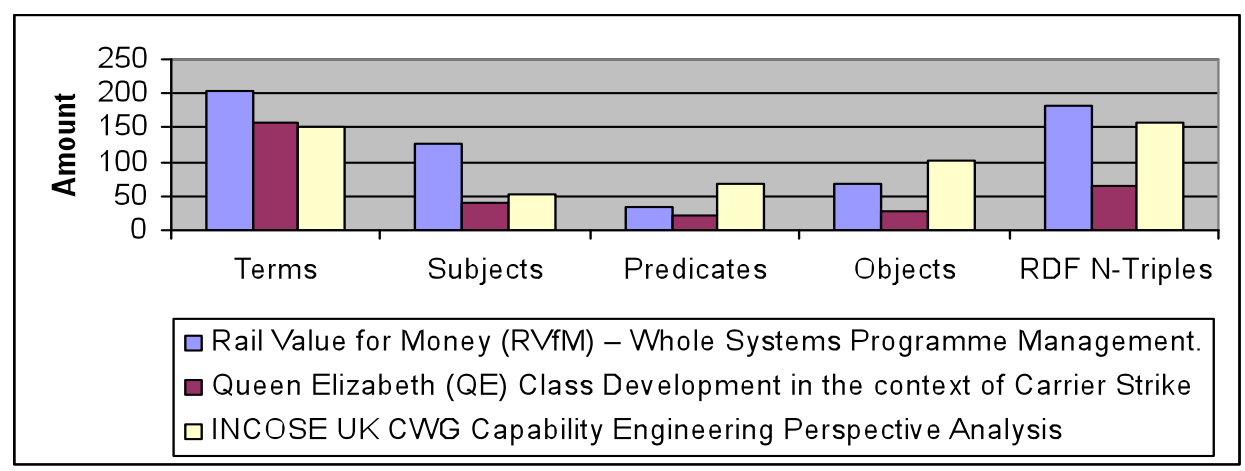

Figure 7. Statistics showing term extraction and n-triples from three case studies

An incremental hybridisation process is used for ontology development, as shown in Figure 8. Each version of the ontology is updated through further case study (CS) analysis and inclusion.

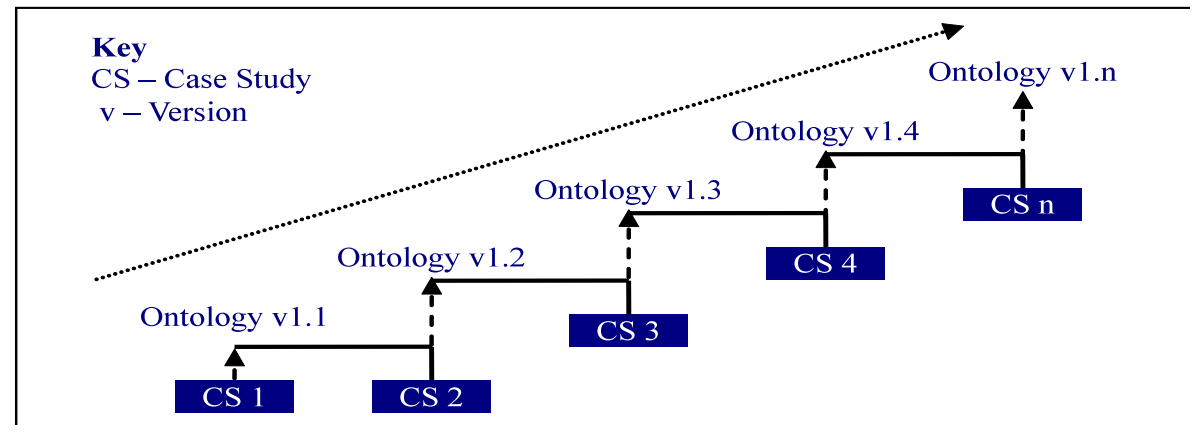

Figure 8. Incremental hybridisation process for ontology development

A capability engineering ontology, referred to as v1.1 in Figure 8, is the first output of this research. This comprises the results from the INCOSE UK CWG perspective analysis case study. The carrier strike capability ontology is still being processed to construct v1.2. The focus group for the rail case study is scheduled for December 2011 and the results will be used to generate v1.3 through incremental hybridisation. Figure 9 shows the initial results for capability engineering ontology v1.1. 


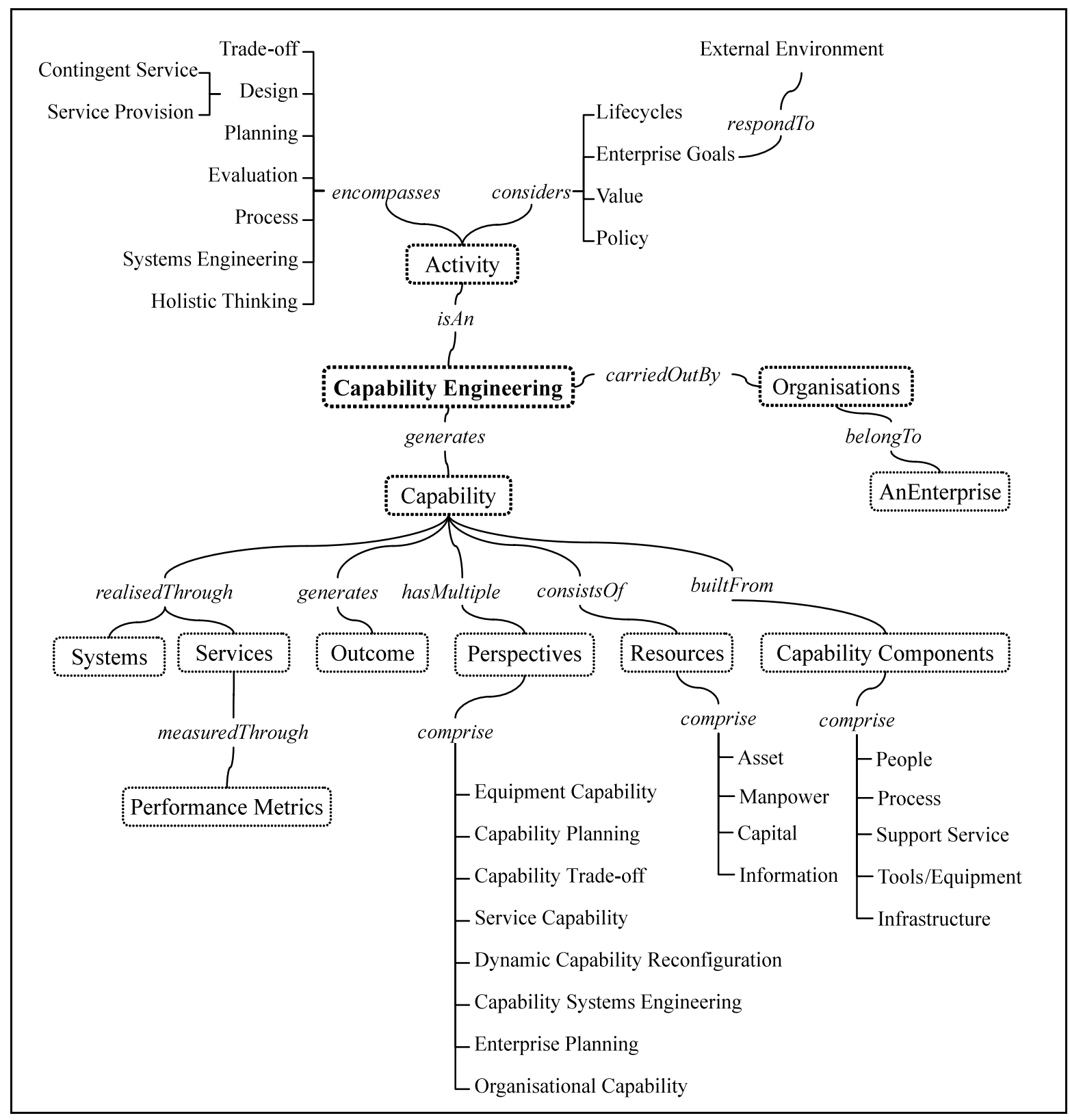

Figure 9. Capability engineering ontology v1.1 derived from the focus group results

Capability engineering ontology v1.1 can be improved through insertion of cardinality that considers relationships such as 1-1, many-1, 1-many and many-many. For example, the use of 'AnEnterprise' and 'Organisations' can be expressed through the cardinality constraints. There are also similarities between 'capability components' and 'resources' which can be exploited through inclusion of other case studies. Performance metrics are also associated with services whereas the initial findings from the carrier strike capability case study refer to performance metrics as "measures of merit" that correlate to classes such as capability, enterprise and system as well as services. The carrier strike case study denotes that capability is emergent property of the hierarchy that considers systems of systems (SoS), systems and sub-systems. Capability engineering ontology v1.2 will consider such aspects. 


\section{Discussion}

This research proposed a process flow chart for incremental ontology development though using case study material from heterogeneous domains. It also presents and discusses initial findings from a study that develops an ontology for capability engineering. The RDF $\mathrm{n}$-triples results are from three case studies with two of them being validated through expert focus groups. Suggestions for corrections and new concepts have been included in the revised list of $n$-triples. The capability engineering ontology presented in Figure 9 is derived from only one case study. An incremental hybridisation process will be employed to derive an updated version of the ontology through inclusion of other case studies.

The limitations of this ontology development include subjectivity which is to some extent minimised through analysing the n-triples i.e. subject-predicate-object clauses via focus groups and expert reviews. Another drawback includes the task of importing the results into Protégé. This task is partly automated but predicates such as 'subClassOf', 'consistsOf', 'comprise' and 'encompasses' require further human assisted analysis for insertion into Protégé. The final ontology will include a definitions list and relationship statements describing each term and subject-predicate-object clause. These can be derived from the MS Excel analysis that already generated statements showing the relationship and context of use of the terms. The evolution process that involves revising the ontology to keep it up to date through i.e. adding, deleting and modifying the ontology is to be tackled when importing into Protégé. An international perspective i.e. seeking feedback from overseas is also planned.

The term extraction tasks that involved using a mark-up tool to highlight the key terms from the case study material was human assisted. It is relatively difficult to implement an automated tool to extract terms as domain knowledge relevant to capability engineering has an important influence on analysis. The final version of the ontology can be an enabler to develop such tools. It is also significantly important to emphasise that the ontology is not complete. For example in v1.1, it is stated that capability engineering is an activity that considers lifecycles, enterprise goals, value, policy etc. This is only derived from a single case study and certainly there may be other considerations that include, for example, governance as this is associated with most activities.

The three phases of the process flow chart i.e. pre-analysis, modelling and post-analysis are very similar to approaches already discussed. The aim of this research was not to validate the proposed flow chart, but rather, to use it to develop an ontology for capability engineering.

\section{Conclusion}

This paper presents the first steps towards establishing an ontology to enable semantic interoperability in order to support institutionalisation of the concept of capability engineering. The task of coming up with a common set of concepts, properties and relationships to enable a standard domain terminology and common understanding within heterogeneous domains is significant, because this will be an enabler of cross-domain knowledge sharing. The capability engineering paradigm is gaining attention in several domains and signals increased efforts to manage large and complex systems more effectively from the acquisition and operational perspectives. The need for greater cross-domain knowledge sharing has never been greater. The remaining challenges for this work include getting general acceptance and development of a hierarchy. However, as illustrated in this paper, analysis of real life case study material and enabling user involvement can tackle such problems. The maturity of the capability engineering ontology is to be improved through 
inclusion of a wider range of case studies and planned validation focus groups. The final version of the ontology will be imported into Protégé to support collaborative discussions i.e. seeking international feedback over the internet. The ultimate goal is to develop a single shared ontology through incremental hybridisation of heterogeneous case studies.

\section{References}

Allemang, D., and J. Hendler. 2008. Semantic Web for the Working Ontologist: Effective Modeling in RDFS and OWL. Morgan Kaufman

Annamalai, G., R. Hussain, M. Cakkol, R. Roy, S. Evans, and A. Tiwari. 2011. "An Initiative to a Product-Service Ontology" CIRP IPS2 Conference, 5-6 May.

Corcho, O., M. Fernandez-Lopez., and A. Gomez-Perez. 2002. "Methodologies, tools and languages for building ontologies: where is their meeting point?" Data and Knowledge Engineering, 46 (1): 41-64.

Dongmin, Z., H. Dachao., and X. Yuchun. 2010. "A framework for ontology-based product design knowledge management". IEEE International Conference on Fuzzy Systems and Knowledge Discovery, Yantai, China.

Duan, J., L. Aiping., and L. Xuemei. 2009. "Ontology-based modelling of structural knowledge for modular mechanical products". IEEE International Conference on Intelligent Computing and Intelligent Systems, Shanghai, China.

Elphick, J. 2011. "Rail Value for Money Study - Whole System Programme Management", DfT and the ORR, Contract number RVFM 10004. Issue 1.4.

Henshaw, M., D. Kemp, P. Lister, A. Daw, A. Harding, A. Farncombe, and M. Touchin. 2010. "Capability Engineering - an analysis of perspectives". INCOSE UK Capability Working Group, Perspectives Analysis Sub-Group (PASG). UK.

Hughes, J. S., D. J. Crichton, and C. A. Mattmann. 2009. "Ontology-based information model development for science information reuse and integration", IEEE International Conference on Information Reuse and Integration, Nevada, USA.

Johnson, P. 2009. "Evaluating Systems of Systems against Mission Requirements" PhD diss., Loughborough University (Loughborough, Leicestershire, UK).

Kourlimpinis, G., G. Gyftodimos, J. Psarras, and D. Askounis. 2008. "Knowledge exploitation through modelling navigation and query, an ontology-based integrated architecture". Pan-Hellenic Conference on Informatics, Samos, Greece.

Liu, X., D. Rosen, and Z. Yu. 2010. "Ontology based knowledge modelling and reuse approach in product design”. IEEE Int. Conf. Information Reuse and Integration, Nevada, USA.

Mizoguchi, R. 2003. "Tutorial on Ontological Engineering - Part 2: Ontology tools, development and languages". New Generation Computing, Ohmsha and Springer, 22 (1): 61-69.

Mizoguchi, R., and K. Kozaki. 2009. "Ontology Engineering Environments". In: International Handbook on Information Systems. Bernus et al., (Eds), Springer-Verlag, 315-336.

NAO (National Audit Office). 2006. Department of Health: The National Programme for IT in the NHS. London: The Stationery Office.

Noy N.F., and D. L. McGuinness. 2000. Ontology Development 101: A Guide to Creating Your First Ontology, Stanford University.

Protégé. 2011. "The Protégé Ontology Editor and Knowledge Acquisition System”. Accessed September 2011. http://protege.stanford.edul.

Studer, R., V. Benjamins, and D. Fensel. 1998. "Knowledge Engineering: Principles and Methods". Data and Knowledge Engineering, 25(1-2):161-197. 
Tan, G., T. Hao, and Z. Zhong. 2009. "A knowledge modelling framework for intangible cultural heritage based on ontology". Second International Symposium on Knowledge Acquisition and Modeling, Wuhan, China.

Touchin, M., A. Harding, S. Leinster-Evans, I.D. Westphal, D. Hawken, Use of an Activity Model to Underpin Enterprise Evolution in Capability Management, Integrated Enterprise Architecture Conference 2010, 9th/10th March 2010

UKMoD 2012, 22/3/2012, http://www.mod.uk/DefenceInternet/AboutDefence/WhatWeDo/Maritime/CarrierStrike/

Unshold, M., and M. Gruninger. 2004. "Ontologies and Semantics for Seamless Connectivity”. SIGMOD Record, 33 (4): 58-64.

Volere. 2011. "Requirements Specification". Accessed April 2011. http://www.volere.co.ukl.

\section{Acknowledgements}

This paper has been prepared with inputs from INCOSE UK Capability Working Group and Loughborough University Engineering Systems of Systems (ESoS) research group.

\section{Biography}

Huseyin Dogan is an Engineering Doctorate candidate within the Engineering Systems of Systems (ESoS) research group at Loughborough University. His research focuses on "through life enterprise knowledge management" jointly sponsored by EPSRC and BAE Systems. Prior to the EngD programme Huseyin was employed as a Higher Scientist within the Human Factors Department at BAE Systems Advance Technology Centre. Huseyin has an MSc in HCI with Ergonomics from UCL and a BSc in Computer Science from Queen Mary, University of London.

Michael Henshaw is Professor of Systems Engineering at Loughborough University. He leads the ESoS research group and has projects across a range of SoS themes. Professor Henshaw is Academic Director of INCOSE UK and also leads the INCOSE UK Capability Working Group. He is co-chair of the IEEE Technical Committee in Systems of Systems and is a panel member of the Systems Concepts and Integration panel in NATO RTO.

Julian Johnson obtained a BSc in Physics and PhD in Physics (Space Science) at Kings and University College, London, respectively. Spent 8 years as a research scientist at University of London, NASA/MSFC, Alabama, and University of Southampton, UK. Since 1987 he has worked in BAE Systems in technical and management roles in systems process-improvement. Technical Manager on the European SEDRES-1, -2 projects, with involvement in ISO 10303 AP233 development, a data exchange standard. Contributed to the early phases of OMG SysML. Currently an Executive Scientist at the Systems Engineering Innovation Centre (SEIC). Interests in information-centred aspects of systems engineering. 